\title{
The effect of uniaxial pressure on the magnetic anomalies of the heavy-fermion metamagnet $\mathrm{CeRu}_{2} \mathrm{Si}_{2}$
}

\author{
S. R. Saha, H. Sugawara, T. Namiki, Y. Aoki, and H. Sato \\ Department of Physics, Graduate School of Science, Tokyo Metropolitan University, \\ Minami-Ohsawa 1-1, Hachioji, Tokyo 192-0397, Japan
}

(Dated: November 2, 2018)

\begin{abstract}
The effect of uniaxial pressure $\left(P_{u}\right)$ on the magnetic susceptibility $(\chi)$, magnetization $(M)$, and magnetoresistance (MR) of the heavy-fermion metamagnet $\mathrm{CeRu}_{2} \mathrm{Si}_{2}$ has been investigated. For the magnetic field along the tetragonal $c$ axis, it is found that characteristic physical quantities, i.e., the temperature of the susceptibility maximum $\left(T_{\max }\right)$, the pagamagnetic Weiss temperature $\left(\Theta_{p}\right), 1 / \chi$ at $2 \mathrm{~K}$, and the magnetic field of the metamagnetic anomaly $\left(H_{M}\right)$, scale approximately linearly with $P_{u}$, indicating that all the quantities are related to the same energy scale, probably of the Kondo temperature. The increase (decrease) of the quantities for $P_{u} \| c$ axis ( $P_{u} \| a$ axis) can be attributed to a decrease (increase) in the nearest $\mathrm{Ce}-\mathrm{Ru}$ distance. Consistently in $\mathrm{MR}$ and $\chi$, we observed a sign that the anisotropic nature of the hybridization, which is believed to play an important role in the metamagnetic anomaly, can be controlled by applying the uniaxial pressure.
\end{abstract}

PACS numbers: $75.20 . \mathrm{Hr}, 71.27 .+\mathrm{a}, 74.62 . \mathrm{Fj}$

\section{INTRODUCTION}

The compound, $\mathrm{CeRu}_{2} \mathrm{Si}_{2}$, crystallizing in the tetragonal $\mathrm{ThCr}_{2} \mathrm{Si}_{2}$-type structure, is one of the most intensively studied and best characterized systems among the heavy-fermion compounds 1 The reason is that apart from a large electronic specific-heat coefficient $\gamma \sim 360 \mathrm{~mJ} / \mathrm{mol} \mathrm{K}{ }^{2}$ in zero field, 2 3 there is an abrupt nonlinear increase of magnetization, the so called metamagnetic anomaly (MA) from a paramagnetic ground state, around an external magnetic field of $H_{M} \sim 80 \mathrm{kOe}$ (Ref. 1) applied only along the $c$ axis of the tetragonal structure below $15 \mathrm{~K}$. Not only the magnetization process but also many other physical properties have been reperted to be anomalous in this field region around $H_{M}$. 3.4 . 5

The origin of the anomaly is still a matter of debate, despite intensive investigations. The de Haas-van Alphen effect studies have shown that both the Fermi surface and the effective mass change considerably around $H_{M}$, suggesting a change of $4 f$-electron character from itinerance in the low-field state to localization in the high-field state 4 In contrast, low-temperature magnetization measurements 5 suggest that the low-field state is continuously connected to the high-field state across $H_{M}$. Moreover, a peak observed in the Hall resistivity at $H=H_{M}$ disappears on approaching $T=\varnothing$, suggesting no abrupt change in the Fermisurface. 6 Reports of hydrostatic pressure experiments 18 reveal that the volume reduction enhances the characteristic energy of the quasiparticle system and leads to a drastic shift of $H_{M}$ to higher fields. From this large effect of pressure a very large electronic Grüneisen parameter $185 \mathrm{Mbar}^{-1}$ has been inferred. The anisotropic hybridization between $4 f$ and conduction electrons leading to an anomalous peak in the quasiparticle density-of-states (DOS) is argued to play an important role in the MA; the MA appears when the peak crosses the Fermi level at high magnetic fields.3. 9 However, no direct evidence of the anisotropic hybridization effect can be obtained from hydrostatic pressure experiments. The uniaxial-pressure experiment has the potential for providing useful information on anisotropic hybridization. In view of these reasons, we have investigated the effect of uniaxial pressure in $\mathrm{CeRu}_{2} \mathrm{Si}_{2}$ with magnetic and transport experiments.

\section{EXPERIMENT}

Single crystals of $\mathrm{CeRu}_{2} \mathrm{Si}_{2}$ were grown by the Czochralski pulling method in a tetra-arc furnace with an argon atmosphere. The single crystalline nature was confirmed by back-reflection-Laue techniques. The high quality of the single crystal was inferred from the residual resistivity ratio $\geq 110$. Electrical resistivity and magnetoresistance were measured by the standard dc four probe method using a computer-controlled current source and nanovoltmeter (182 Keithley), using a top-loading ${ }^{3} \mathrm{He}$ cryostat equipped with a $160 \mathrm{kOe}$ superconducting magnet (Oxford Instruments Co., Ltd.). The electrical contacts of Ag current leads were affixed to the sample by indium soldering. Au wires of $80-\mu \mathrm{m} \phi$ were spotwelded to the sample as voltage leads. Uniaxial pressures were generated by using a piston-cylinder-type $\mathrm{CuBe}$ pressure cell for transport experiments, recently designed and constructed by us 10 , so as to suit the above cryostat. Single-crystal samples (typical dimensions: $\sim 0.7 \times 1 \times 2 \mathrm{~mm}^{3}$ ) were sandwiched between two disc-shaped $\mathrm{ZrO}_{2}$ plates, which provided the electrical isolation. A Nitflon tape of $0.08-\mathrm{mm}$ thickness was placed between the sample and $\mathrm{ZrO}_{2}$ plates in order to prevent the breaking of the sample due to its surface roughness under pressure, if any. Uniaxial pressures were 
applied on a $\mathrm{ZrO}_{2}$ ball, placed inside a ring-shaped guide on the $\mathrm{ZrO}_{2}$ plate above the sample, which prevented any rotation of the sample under pressure. Uniaxial pressures produced on the sample at low temperatures were calibrated by measuring the superconducting transition temperature of Sn placed in the cell by an induction method. Magnetic properties were measured by a commercial superconducting quantum interference device (SQUID) magnetometer. Uniaxial pressures parallel to the magnetic fields were generated by using a modified version of the SQUID-pressure cell reported by Uwatoko et al 11 Uniaxial pressures produced on the rectangular shaped single crystal $(\sim 1.5 \times 1.5 \times 2$ $\mathrm{mm}^{3}$ ) at low temperatures were calibrated by measuring the Meissner effect of a small piece of $\mathrm{Pb}$, placed in the pressure cell. The known pressure dependences of the superconducting transition temperature of Sn (Ref. 12) (for transport measurements) and $\mathrm{Pb}$ (Ref. 13) were used for these purposes. Uniaxial pressures perpendicular to the magnetic fields were generated by a uniaxial-pressure cell recently designed and constructed by us 10 . In this case, the uniaxial pressures were determined at room temperature by the absolute value of force applied on the sample. The total magnetization of the sample and the uniaxial-pressure cell were measured by SQUID magnetometer and the magnetization of the uniaxial-pressure cell, though small $(1-10 \%)$, were subtracted from the total magnetization to obtain the precise value of the sample magnetization.

\section{EXPERIMENTAL RESULTS}

Figure 11 shows the effect of uniaxial pressure on the temperature dependence of magnetic susceptibility $\chi(T)$ in $\mathrm{CeRu}_{2} \mathrm{Si}_{2}$; Fig. 11(a) is for $P_{u}\|H\| c$ axis, Fig. 11(b) is for $P_{u} \| a$ and $H \| c$ axis, and Fig. 11(c) is for $P_{u}\|H\| a$ axis. At ambient pressure, the magnetic susceptibility is strongly anisotropic depending on whether $H$ is applied parallel or perpendicular to the tetragonal $c$ axis. $\chi_{H \| c}(T)$ obeys the Curie-Weiss law above $\sim 70 \mathrm{~K}$ and then shows a maximum at a temperature $T_{\max } \simeq 10 \mathrm{~K}$, which is considered to provide a measure of the Kondo temperature $T_{\mathrm{K}} \cdot \chi_{H \| a}(T)$ also follows the Curie-Weiss law above $100 \mathrm{~K}$, and it exhibits much smaller values than $\chi_{H \| c}(T)$, without showing any maximum. The anisotropy ratio $\chi_{H \| c}(T) / \chi_{H \| a}(T)$ increases largely with decreasing temperatures. These behaviors at ambient pressure are consistent with those reported before 7 . When the uniaxial pressure of $P_{u} \| c$ axis is applied, $\chi_{H \| c}^{P_{u} \| c}$ for $H \| c$-axis is largely suppressed at low temperatures with increasing $P_{u}$, though the effect of $P_{u}$ is small at higher temperature $(\geq 100 \mathrm{~K})$. $T_{\max }$ shifts to higher temperatures with increasing $P_{u}$, i.e., $T_{\max } \simeq 16.5$ $\mathrm{K}$ at $P_{u} \simeq 3.27 \mathrm{kbar}$. The pressure dependence is almost similar to that under hydrostatic pressure $\left(P_{h}\right)$ repoted in Refs. 7 and 8. By contrast, when $P_{u}$ is applied per-
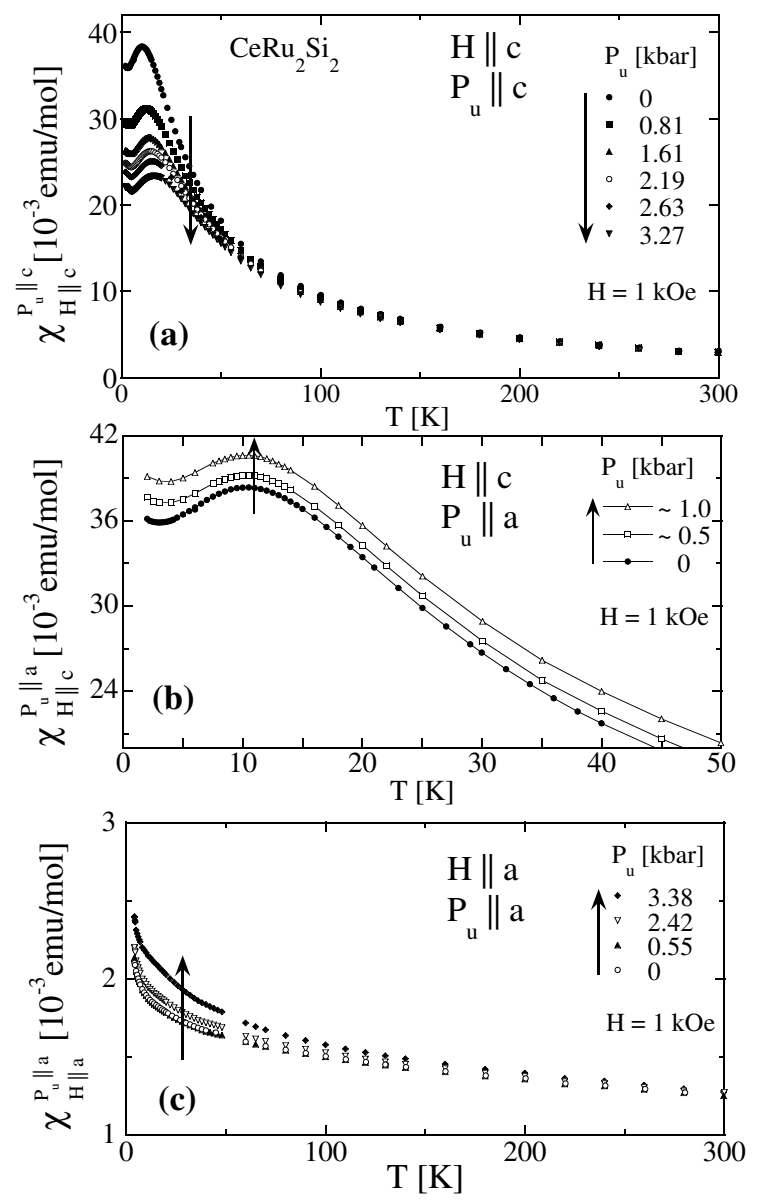

FIG. 1: Temperature dependence of magnetic susceptibility $(\chi)$ in $\mathrm{CeRu}_{2} \mathrm{Si}_{2}$ under uniaxial pressure $\left(P_{u}\right)$. (a) $\chi_{H \| c}^{P_{u} \| c}(T)$ for $P_{u}$ applied parallel with the magnetic field $(H)$ along the tetragonal $c$ axis. (b) $\chi_{H \| c}^{P_{u} \| a}(T)$ for $P_{u}$ along the $a$ axis and $H$ along the $c$ axis. (c) $\chi_{H \| a}^{P_{u} \| a}$ for $P_{u}$ and $H$ along the $a$ axis. The arrows indicate the change of $\chi$ with increasing $P_{u}$.

pendicular to the magnetic field, i.e., $P_{u} \| a$ axis, $\chi_{H \| c}^{P_{u} \| a}$ at low temperatures is enhanced and $T_{\max }$ is slightly suppressed [see Fig. 1 (b)]. On the other hand for $H \| a$ axis, $\chi_{H \| a}^{P_{u} \| a}$ slightly increases at low temperatures with $P_{u} \| a$ axis [see Fig. 11(c)]. This increase of $\chi_{H \| a}^{P_{u} \| a}$ is also in contrast to the decrease of $\chi_{H \| a}^{P_{h}}$ under hydrostatic pressure In the case of $P_{u} \| c$ axis, $\chi_{H \| a}^{P_{u} \| c}$ weakly enhances at low temperatures with $P_{u}$ (not shown); it is difficult to separate accurately the background contribution from the pressure cell which is comparable to the sample magnetization for $H \| a$ axis.

Figure 2 shows the effect of uniaxial pressure on the magnetic-field dependence of isothermal (at $2 \mathrm{~K}$ ) magnetization $M(H)$ in $\mathrm{CeRu}_{2} \mathrm{Si}_{2}$. The ambient pressure data are consistent with those reported befored. For $H \| c$ axis, a positive curvature of $M_{H \| c}^{P_{u} \| c}(H)$ is observed as a 


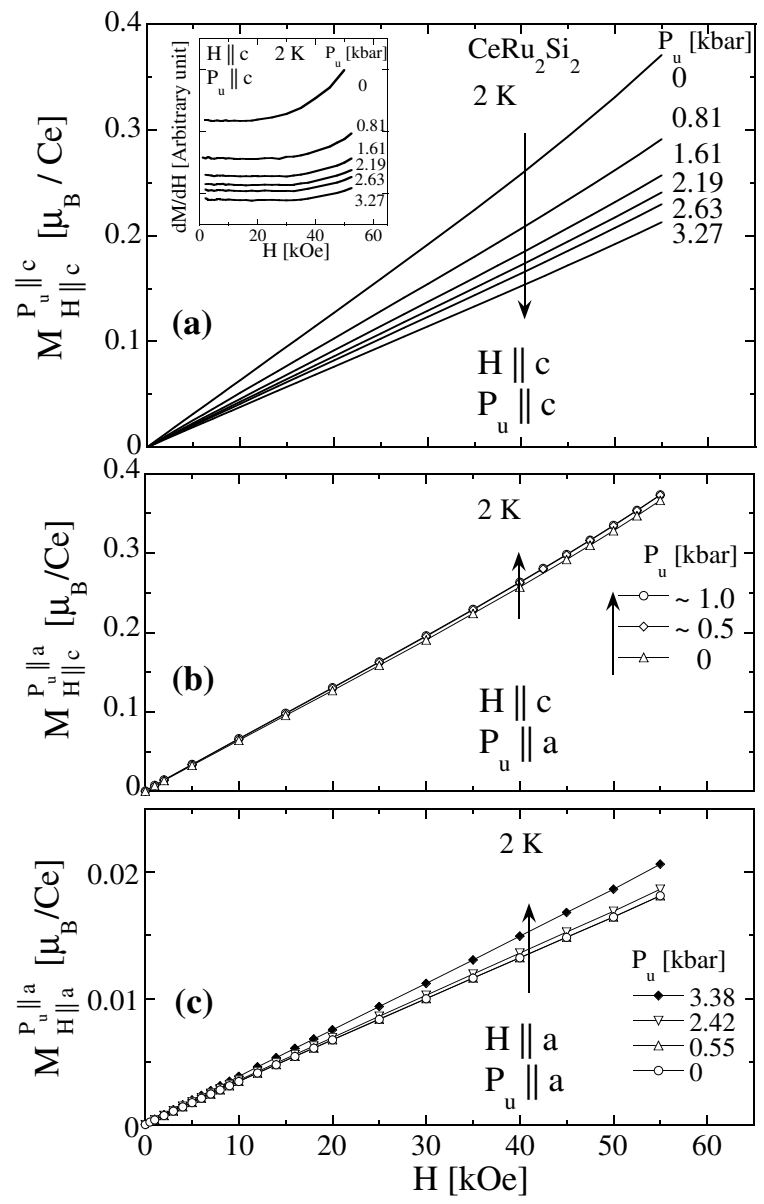

FIG. 2: Magnetic-field dependence of isothermal magnetization $M(H)$ in $\mathrm{CeRu}_{2} \mathrm{Si}_{2}$ under uniaxial pressure at $2 \mathrm{~K}$. (a) $M_{H \| c}^{P_{u} \| c}(H)$ for $P_{u}$ applied parallel with the magnetic field along the tetragonal $c$ axis. (b) $M_{H \| c}^{P_{u} \| a}(H)$ for $P_{u}$ along the $a$ axis and $H$ along the $c$ axis. (c) $M_{H \| a}^{P_{u} \| a}(H)$ for $P_{u}$ and $H$ along the $a$ axis. The arrows indicate the change of $M$ with increasing $P_{u}$. The solid lines are guides to the eyes.

precursor to the metamagnetic anomaly at $H_{M} \simeq 80 \mathrm{kOe}$ at ambient pressure. Under $P_{u} \| c$ axis, $M_{H \| c}^{P_{u} \| c}$ up to 55 kOe drastically decreases [see Fig. 2(a)] and the curvature $\partial M_{H \| c}^{P_{u} \| c} / \partial H$ at $55 \mathrm{kOe}$ is also largely suppressed with increasing $P_{u}$ as shown in the inset of Fig. 2(a). These facts indicate a shift of the metamagnetic anomaly to higher magnetic fields by $P_{u} \| c$ axis; actually, this is confirmed by the magnetoresistance measurement (see Fig. 3). This effect of $P_{u} \| c$ axis on the isothermal $M_{H \| c}^{P_{u} \| c}(H)$ is similar to the effect of $P_{h}$ on the isothermal magnetization $M_{H \| c}^{P_{h}}(H)$ reported in Ref. 7, where $M_{H \| c}^{P_{h}}$ and $\partial M_{H \| c}^{P_{h}} / \partial H$ also drastically decrease with increasing $P_{h}$. By contrast, under $P_{u} \| a$ axis, $M_{H \| c}^{P_{u} \| a}$ is enhanced [see Fig. 2(b)] and the $\partial M_{H \| c}^{P_{u} \| a} / \partial H$ at $55 \mathrm{kOe}$ is also weakly enhanced. On the other hand for $H \| a$ axis, $P_{u} \| a$ axis enhances $M_{H \| a}^{P \| a}$ [see Fig. 2(c)]. This increase of $M_{H \| a}^{P_{u} \| a}$ is in sharp contrast to the decrease of $M_{H \| a}^{P_{h}}$ under hydrostatic pressuret.

In order to see the effect of uniaxial pressure on

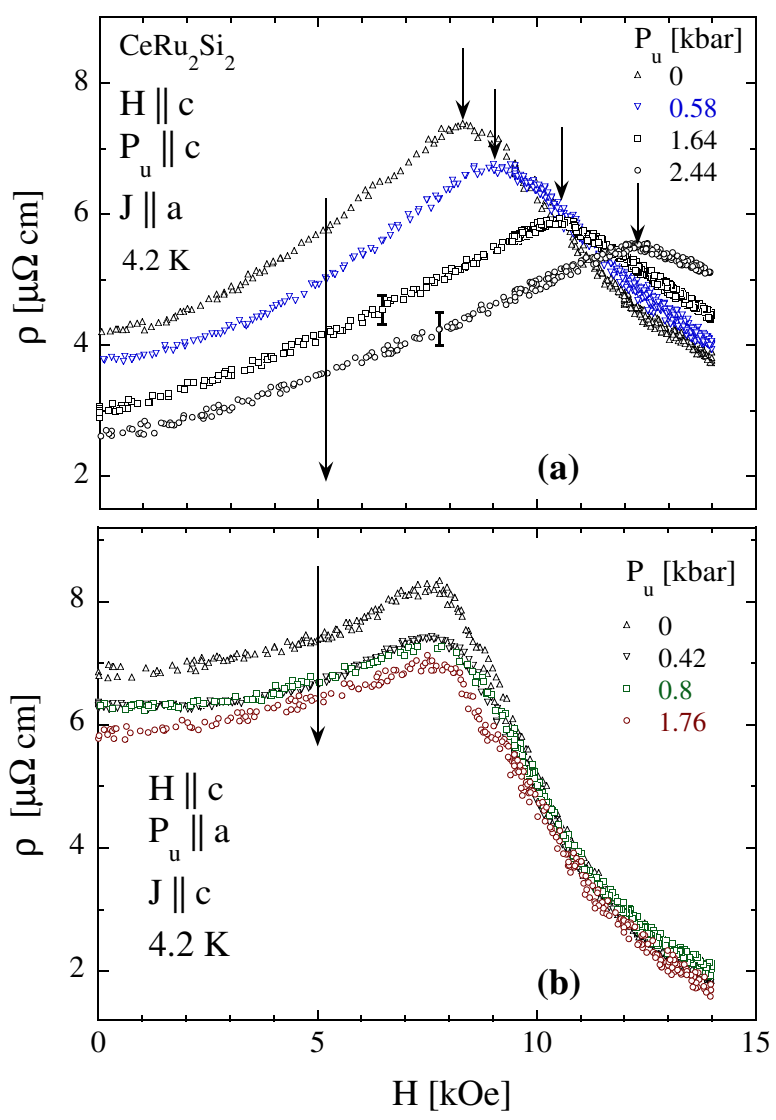

FIG. 3: Magnetoresistance (MR) in $\mathrm{CeRu}_{2} \mathrm{Si}_{2}$ at $4.2 \mathrm{~K}$ under uniaxial pressure $P_{u}$ (a) for $P_{u} \| c$ axis $\left(0 \leq P_{u} \leq 2.44 \mathrm{kbar}\right)$ and transverse geometry ( $H \| c$ axis, $J \| \bar{a}$ axis). The short arrows indicate the magnetic fields $H_{M}$ where the maximum in $\rho(H)$ occurs, reflecting the metamagnetic anomaly. The error bars are put in the vertical axis after smoothing the scattered data points at higher pressures due to deterioration of the spotweld of the voltage leads. (b) for $P_{u} \| a$ axis (0 $\left.\leq P_{u} \leq 1.76 \mathrm{kbar}\right)$ and longitudinal geometry $(H\|J\| c$ axis). The long arrows indicate the decrease of $\rho$ below $H_{M}$ with increasing $P_{u}$ in both figures.

the MA, we have measured the magnetoresistance (MR) up to $140 \mathrm{kOe}$ under $P_{u}$. Figure 3(a) shows the effect of $P_{u} \| c$ axis on the magnetic field dependence of the transverse magnetoresistance, i.e., $H$ along the $c$ axis and the current $J$ along the $a$ axis. The data for $P_{u} \sim 0 \mathrm{kbar}$ are in close agreement with that reported in Ref. 8. The resistivity increases with magnetic fields and then shows a maximum or peak at $H_{\max } \simeq 83 \mathrm{kOe}$. The MA manifests 
itself in this peak at $H_{\max }\left(\equiv H_{M}\right)$. With increasing $P_{u}$, the resistivity below $H_{M}$ decreases and the peak shifts to higher $H$ as marked by vertical arrows. These behaviors are similar to those under hydrostatic pressure reported in Ref. 8, though there are small quantitative differences. The most significant differences are that the absolute value of $\rho$ at the peak (at $H_{M}$ ) decreases and the shape of the peak broadens with increasing $P_{u} \| c$-axis, while these remain almost unchanged for all values of $P_{h}$. These behaviors under $P_{h}$ have been ascribed to the implication that the quasiparticle DOS always reaches the same critical value at $H_{M}$. This conclusion was made by taking into account the following facts: $H_{M}$ depends on pressure but it depends only slightly on temperature. At all values of $P_{h}$, the $\rho$ at $H=H_{M}$ of the corresponding $P_{h}$ shows almost the same temperature dependence. Therefore, the slope of each $\rho(T)$ curve of $H=H_{M}$, which gives an estimation apout the DOS at $H=H_{M}$, remains unchanged with $P_{h}$. Based on the same model, in the present case under $P_{u} \| c$ axis, the decrease and broadening of the peak at $H_{M}$ may indicate a change of the quasiparticle DOS near the metamagnetic anomaly at $H_{M}$ with increasing $P_{u} \| c$ axis. However, measurements at low temperatures are necessary in order to reach a decisive conclusion.

Figure 3(b) shows the effect of $P_{u} \| a$ axis on the magnetic field dependence of longitudinal magnetoresistance, i.e., $H$ along $c$ axis and $J$ along the $a$ axis. The application of $P_{u} \| a$ axis is very risky, since large cracks easily develop into the samples. Some samples were completely separated into pieces in the $c$ plane under pressure, making the measurement impossible several times. The ambient pressure $\left(P_{u} \sim 0\right)$ data cannot be compared with that of Ref. 8, since there is no data for this geometry, however, the data are consistent in nature to the reported longitudinal MR at lower temperatures by Kambe et al. 6 . The data for $P_{u} \sim 0$ and $0.8 \mathrm{kbar}$ have been taken on the same sample piece, but for $P_{u} \sim 0.4$ and $1.76 \mathrm{kbar}$ the data have been taken on two different sample pieces, although all the pieces were cut from the adjacent part of the same crystal. The results shown in Fig. 3(b) indicate that the sample dependence is minor at least for these three pieces. With increasing $P_{u} \| a$ axis, the resistivity decreases for all $H$, though the change in $\rho$ above $H_{M}$ is smaller than that below $H_{M}$. The magnetoresistance peak at $H_{M}(\sim 80 \mathrm{kOe}$ at ambient pressure), manifesting the metamagnetic anomaly, changes only slightly with increasing $P_{u}$. There is a slight decreasing tendency of $H_{M}$ [see Fig. 4(c)] with increasing $P_{u}$, although it is difficult to determine accurately the rate $\Delta H_{M} / \Delta P_{u}$ due to its extremely small change relative to the experimental accuracy.

Figure 4 (a) shows the dependence of $T_{\max }$ in $\chi_{H \| c}(T)$ on $P_{u}$ along with that on $P_{h}$ reported by Voiron et al.. . $T_{\max }$ is enhanced with $P_{u} \| c$ axis as $\mathrm{d} T_{\max } / \mathrm{d} P_{u} \simeq 1.85$ $\pm 0.1 \mathrm{~K} / \mathrm{kbar}$ which is close to the rate $\mathrm{d} T_{\max } / \mathrm{d} P_{h} \simeq 2.5$ $\mathrm{K} / \mathrm{kbar}$ for the $P_{h}$ reported in Refs. 7 and 8. The absolute value of the paramagnetic Weiss temperature $\Theta_{\mathrm{p}}$, de-
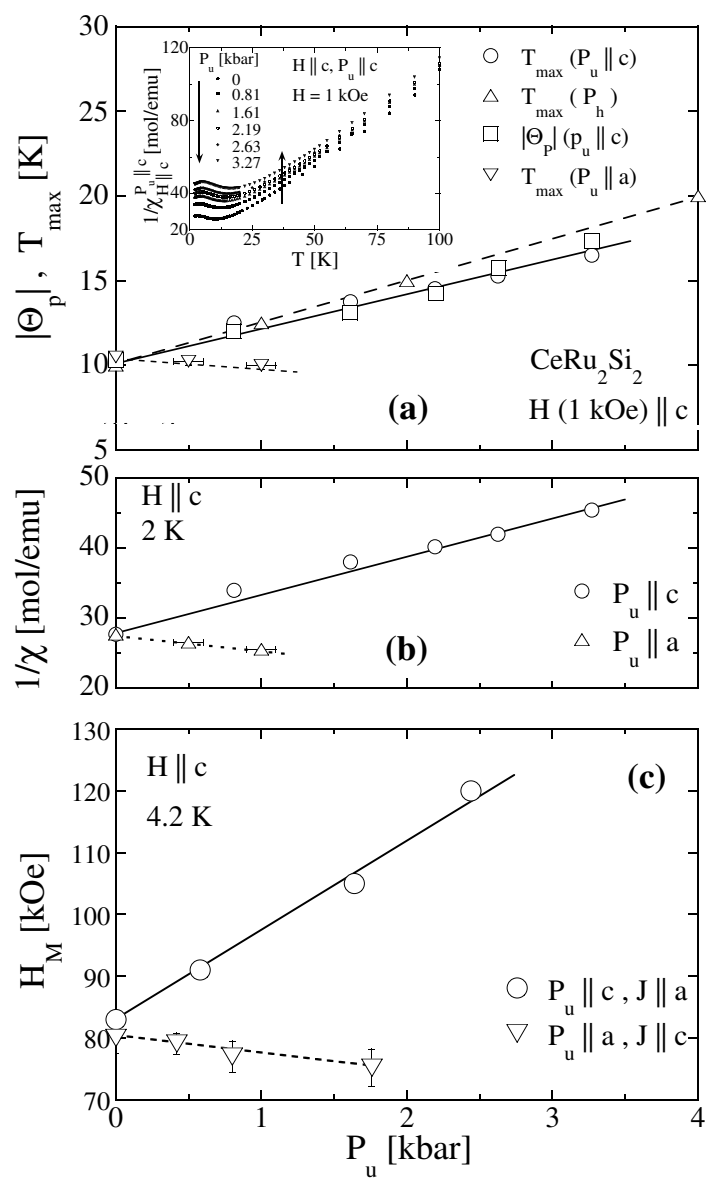

FIG. 4: (a) Uniaxial pressure dependence of $T_{\max }$ in $\chi_{H \| c}^{P_{u} \| c}$ and the absolute value of the paramagnetic Weiss temperature $\Theta_{p}$, estimated from the linear region of the inverse $\chi_{H \| c}^{P_{u} \| c}$ plot below $100 \mathrm{~K}$ shown in the inset; the evolution of $T_{\max }$ under $P_{h}$ reproduced from the report by Voiron et al. (Ref. 7) is also ploted at the same time. (b) Uniaxial-pressure dependence of the inverse $\chi_{H \| c}^{P_{u} \| c}$ and $\chi_{H \| c}^{P_{u} \| a}$ at $2 \mathrm{~K}$. (c) Uniaxial pressure dependence of $H_{M}$ in the transverse ( $H \| c$ axis, $J \| a$ axis) magnetoresistance under $P_{u} \| c$-axis and longitudinal $(H \|$ $J \| c$ axis) magnetoresistance under $P_{u} \| a$ axis in $\mathrm{CeRu}_{2} \mathrm{Si}_{2}$. The solid and broken lines are guides to eyes.

termined from the linear region of the inverse of $\chi_{H \| c}^{P_{u} \| c}(T)$ plot (below $100 \mathrm{~K}$ ) shown in the inset of Figure 4 (a), is also found to increase with $P_{u} \| c$ axis as shown in the same figure. The negative sign of $\Theta_{p}$ can be attributed to Kondo correlations. On the other hand, $T_{\max }$ is suppressed by $P_{u} \| a$ axis. Fig. 1 (b) shows the $P_{u}$ dependence of $1 / \chi_{H \| c}$ at $T=2 \mathrm{~K}$, which is enhanced and suppressed by $P_{u} \| c$ axis and $P_{u} \| a$ axis, respectively. Note that the low-temperature value of $\chi^{-1}$ is proportional to the Kondo temperature $T_{K}$ in the Fermi-liquid regime. Figure 4 (c) shows the $P_{u}$ dependence of $H_{M}$ in MR. $H_{M}$ strongly increases by $P_{u} \| c$ axis as $\Delta H_{M} / \Delta P_{u} \sim 17 \pm 2$ $\mathrm{kOe} / \mathrm{kbar}$ which is close to the rate $\sim 20 \mathrm{kOe} / \mathrm{kbar}$ under 
$P_{h}$ 目, while $H_{\text {畹 }}$ slightly decreases by $P_{u} \| a$ axis.

Mignot et al.8 have shown that the hydrostatic pressure dependence of $T_{\max }, \chi_{T \simeq 0}^{-1}$, and $H_{M}$ show a scaling behavior. A similar plot has been made as a function of $P_{u}$, where all the quantities $T_{\max }, \Theta_{\mathrm{p}}, \chi_{T \simeq 0}^{-1}$, and $H_{M}$ are normalized by their ambient pressure values as shown in Fig. 5. $T_{\max }$ under $P_{h}$ normalized by the ambient pressure value is also plotted for comparison. It is clear that all the quantities scale roughly falling on the same lines. This fact indicates, similar to the case of hydrostatic pressure 8 , an existence of a single energy parameter that determines the low temperature properties controlled by $P_{u}$. Considering that both the low-temperature value of $\chi_{T \simeq 0}^{-1}$ in the Fermi-liquid regime and $\Theta_{p}$ are proportional to the Kondo temperature $T_{K}$, the observed scaling strongly indicates that both $T_{\max }$ and $H_{M}$ are also related to the Kondo effect. The strong increase (weak decrease) of all the quantities indicates the strong enhancement (the weak suppression) of hybridization between conduction and $f$ electrons under $P_{u} \| c$ axis $\left(P_{u} \| a\right.$ axis). In other words, $T_{K}$ is strongly increased by $P_{u} \| c$ axis, while it is decreased by $P_{u} \| a$ axis.

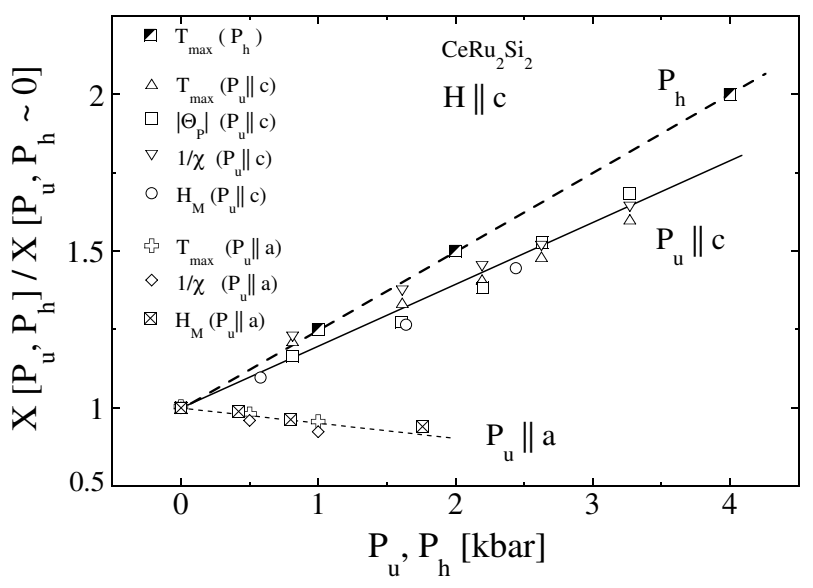

FIG. 5: $T_{\max }, \Theta_{p}, \chi_{T \simeq 0}^{-1}$, and $H_{M}$ normalized by the ambient pressure value of each parameter as a function of $P_{u}$. Solid lines are drawn to show that all the normalized parameters $(X)$ for the same uniaxial-pressure geometry fall roughly on the same line. $T_{\max }$ under $P_{h}$, reported in Ref. 7 normalized by the ambient pressure value is also ploted at the same time. The solid and broken lines are guides to the eyes.

\section{DISCUSSIONS}

In the previous section, we have shown that the effect of uniaxial pressures on the transport and magnetic properties is quite anisotropic. Especially, a qualitatively opposite effect on $T_{K}$ is observed between $P_{u} \| a$ axis and $P_{u} \| c$ axis configurations. In order to understand this, we estimate the movement of the surrounding ions relative to the Ce ions due to the uniaxial pressures, taking into account the elastic properties reported to date.

The elastic constants of $\mathrm{CeRu}_{2} \mathrm{Si}_{2}$ at $300 \mathrm{~K}$ have been reported by Weber 14 , i.e., $\mathrm{C}_{11} \simeq 2.145, \mathrm{C}_{12} \simeq 0.67$, $\mathrm{C}_{33} \simeq 1.215$, and $\mathrm{C}_{13} \simeq 0.806 \mathrm{Mbar}$. We use these values since the low temperature values are not complete; no data are available at low temperatures for $\mathrm{C}_{12}$ in Ref. 14. Note that the temperature dependence of these quantities does not qualitatively affect our consequence shown below. Using these values, the linear compressibilities along the principal crystalline directions are then calculated as $\kappa_{a} \equiv-\left[\Delta a / \Delta P_{u}\right] / a \simeq 0.63 \mathrm{Mbar}^{-1}$ and $\kappa_{c} \equiv-\left[\Delta c / \Delta P_{u}\right] / c \simeq 1.33 \mathrm{Mbar}^{-1}$ for the $a$ axis and $c$ axis, respectively. Three Poisson ratios are also calculated. Under $P_{u} \| a$ axis, the Poisson ratio along the $c$ axis is $\nu_{c}^{P_{u} \| a} \simeq 0.608$, while that along another $a$ axis is $\nu_{a}^{P_{u} \| a} \simeq 0.084$. On the other hand under $P_{u} \| c$ axis, the Poisson ratio is $\nu_{a}^{P_{u} \| c} \simeq 0.286$. The values are quite anisotropic, i.e., for $P_{u}$ along the $a$ axis, the elongation along the $c$ axis is more than seven times larger than that along the other $a$ axis.

It is believed that the hybridization between $f$ states and conduction electrons in $\mathrm{CeRu}_{2} \mathrm{Si}_{2}$ is mainly governed by $d-f$ hybridization 15 . The band-structure calculation 16 indicates that there are five bands crossing the Fermi level (four hole sheets and one electron sheet), and they consist dominantly of the $\mathrm{Ce} 4 f$ and the $\mathrm{Ru} 4 d$ components. Actually, the nearest atom from Ce ions is $\mathrm{Ru}$, suggesting strong hybridization between $4 f$ and $4 d$ electrons. Using the same lattice constants and atomic positions used in Ref. 16, the distances between the nearest neighbor $\mathrm{Ce}-\mathrm{Ru}$ are calculated as a function of uniaxial pressure. The results are shown in Fig. 6. For the cases of applied uniaxial pressure along the $c$-axis and hydrostatic pressure, $\mathrm{Ce}-\mathrm{Ru}$ distance $d_{\mathrm{Ce}-\mathrm{Ru}}$ (= $3.224 \AA$ at ambient pressure) decreases. On the other hand for the uniaxial pressure along the $a$ axis, the crystal symmetry decreases from tetragonal to orthorhombic and two unequivalent $d_{\mathrm{Ce}-\mathrm{Ru}}$ appear, however, the average value of $d_{\mathrm{Ce}-\mathrm{Ru}}$ weakly increases. In the former case, decreasing $d_{\mathrm{Ce}-\mathrm{Ru}}$ should cause an increase of the $d-f$ exchange interaction $J_{d f}$, and also an increase of $T_{K}$, which depends on $J_{d f}$ essentially as $\propto \exp \left[-1 / J_{d f} N\left(E_{F}\right)\right]$, where $N\left(E_{F}\right)$ represents the density of states on the Fermi energy and is less sensitive to pressure than $J_{d f}$. On the contrary, the uniaxial pressure along the $a$ axis should decrease $J_{d f}$ and hence $T_{K}$. These expectations are qualitatively consistent with the present observation. Especially, similar behavior observed for the cases of $P_{u} \| c$ axis and hydrostatic pressure experiments are naturally explained as a consequence of the similar pressure dependencies of $d_{\mathrm{Ce}-\mathrm{Ru}}$.

The large anisotropy in the magnetic susceptibility in $\mathrm{CeRu}_{2} \mathrm{Si}_{2}$ is ascribed to the crystalline electric field $(\mathrm{CEF})$ effect. At ambient pressure, the anisotropy 


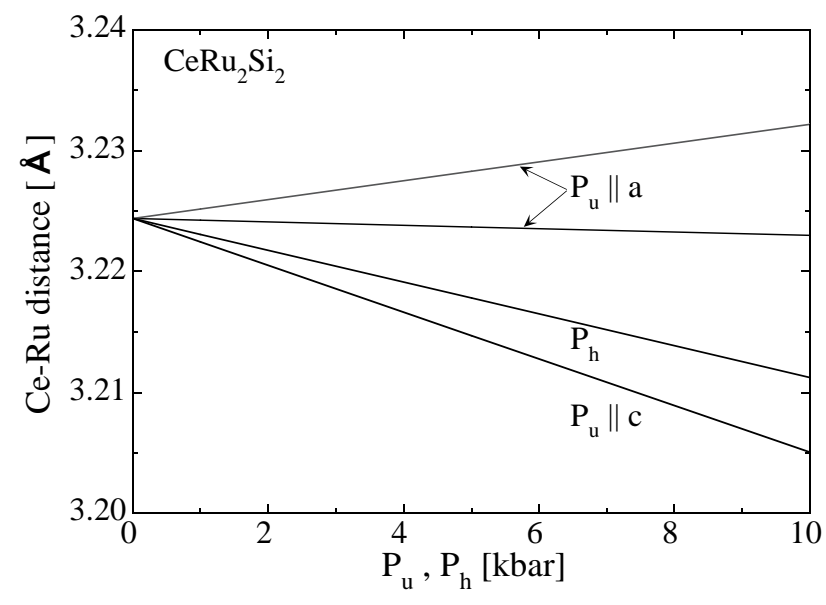

FIG. 6: Estimated change of $\mathrm{Ce}-\mathrm{Ru}$ distance in $\mathrm{CeRu}_{2} \mathrm{Si}_{2}$ with uniaxial and hydrostatic pressure using the values of elastic constants (Ref. 14). The solid lines are guides to the eyes.

$\chi_{H \| c} / \chi_{H \| a} \sim 15$ at $4.2 \mathrm{~K}$ suggests the ground state is $\left.a\left| \pm \frac{5}{2}-\sqrt{\left(1-a^{2}\right)}\right| \mp \frac{3}{2}\right\rangle, a \sim 0.96$ 目 The maximum anisotropy in the magnetic susceptibility is expected for a CEF ground state of pure $\left| \pm \frac{5}{2}\right\rangle . \chi_{H \| c}$ is largely suppressed, while $\chi_{H \| a}$ is slightly enhanced by $P_{u} \| c$ axis. Therefore, the anisotropy $\chi_{H \| c} / \chi_{H \| a}$ decreases with increasing $P_{u} \| c$ axis. This may reflect a change of the CEF ground state, i.e., an increase of the $\left|\mp \frac{3}{2}\right\rangle$ component in the ground state by $P_{u} \| c$ axis. For the $\mathrm{Ce}^{3+}$ ion, the charge distribution of $\left|\mp \frac{3}{2}\right\rangle$ is dumbbell shaped with its elongation along the $c$-axis 27 . Moreover in the $\mathrm{CeRu}_{2} \mathrm{Si}_{2}$ crystal unit cell, the $\mathrm{Ru}$ atom is located at $\left(0, \frac{1}{2}, \pm \frac{1}{4}\right)$ with respect to the Ce ion. 16 Therefore, besides the decrease of $\mathrm{Ce}-\mathrm{Ru}$ distance, an increase of the $\left|\mp \frac{3}{2}\right\rangle$ component also consistently favors the strong enhancement of Ce-Ru hybridization by $P_{u} \| c$ axis. It is argued that there is an anomalous peak structure in the partial density of the hybridized-band state (the DOS) due to anisotropic hybridization between $4 f$ and conduction electrops in the case where the lowest CEF level is $\left| \pm \frac{5}{2}\right\rangle, 3$ In this case, the anisotropic hybridization has an angular dependence characterized by $\left(1-\hat{\mathrm{k}}_{z}^{2}\right)^{2}$. The differential susceptibility diverges when the peak in the DOS crosses the Fermi level giving rise to the metamagnetic anomaly at $H_{M}$. As discussed in the previous section comparing the transverse MR under $P_{u} \| c$ axis with the reported MR behavior under hydrostatic pressure, the decrease and broadening of the $\rho$ peak at $H_{\text {max }}$ may suggest a change of DOS with $P_{u} \| c$ axis. A possible change of the CEF ground state, i.e., an increase of the $\left|\mp \frac{3}{2}\right\rangle$ component by $P_{u} \| c$ axis may cause a decrease and broadening of the anomalous peak in the DOS near the Fermi level predicted for anisotropic hybridization. It may be noted that for a pure $\left|\mp \frac{3}{2}\right\rangle$ ground state the DOS near the Fermi level has a finite value rather than a peales shown in the case of the Kondo insulator CeNiSn 9 18. In order to determine the quantitative change or broadening of the DOS, measurements of the field dependence of specific heat under uniaxial pressure are needed.

\section{CONCLUSION}

We have found that the uniaxial pressure has an anisotropic effect on the magnetic and transport properties in the heavy-fermion metamagnet $\mathrm{CeRu}_{2} \mathrm{Si}_{2}$ with a direct influence on the hybridization. The characteristic parameters $T_{\max }, \Theta_{p}, \chi_{T \simeq 0}^{-1}$, and $H_{M}$ roughly scale as the uniaxial pressure is varied, leading to a single-energy-scale picture, namely, the variation of $T_{K}$. The results suggest that $T_{K}$ (or $d-f$ hybridization) is strongly enhanced for the pressure along the $c$ axis due to the decrease of the nearest $\mathrm{Ce}-\mathrm{Ru}$ distance, while $T_{K}$ is weakly suppressed for the pressure along the $a$ axis due to the increase of $\mathrm{Ce}-\mathrm{Ru}$ distance. The decrease of the anisotropy of magnetic susceptibility and the decrease and broadening of the magnetoresistance peak at the metamagnetic anomaly indicate a controlling of the anisotropic hybridization by uniaxial pressure.

\section{ACKNOWLEDGMENT}

The authors are grateful to thank Profs. E. V. Sampathkumaran, M. Kohgi, O. Sakai, M. Yoshizawa, Y. Uwatoko, and K. Miyake for their comments and help. This work has been partly supported by a Grant-in-Aid for Scientific Research from the Ministry of Education, Science, Sports, and Culture of Japan.
1 P. Haen, J. Flouquet, F. Lapierre, P. Lejay, and G. Remenyi, J. Low. Temp. Phys. 67, 391 (1987).

2 R.A. Fisher, C. Marcenat, N. E. Phillips, P. Haen, F. Lapierre, P. Lejay, J. Flouquet, and J. Voiron, J. Low Temp. Phys. 84, 49 (1991).

3 Y. Aoki, T. D. Matsuda, H. Sugawara, H. Sato, H. Ohkuni, R. Settai, Y. Ōnuki, E. Yamamoto, Y. Haga, A. V. Andreev, V. Sechovoský, L. Havela, H. Ikeda, and K. Miyake
J. Magn. Magn. Mater. 177-181, 271 (1998).

${ }^{4}$ H. Aoki, S. Uji, A. K. Albessard, and Y. Ōnuki, Phys. Rev. Lett. 71, 2110 (1993).

5 T. Sakakibara, T. Tayama, K. Matsuhira, H. Mitamura, H. Amitsuka, K. Maezawa, and Y. Ōnuki, Phys. Rev. B 51, R12 030 (1995).

${ }^{6}$ S. Kambe, J. Flouquet, P. Haen, and P. Lejay, J. Low Temp. Phys. 102, 477 (1996). 
7 J. Voiron, J.-M. Mignot, P. Haen, and J. Flouquet, J. Phys. (France) 49, 1555 (1988).

8 J.-M. Mignot, A. Ponchet, P. Haen, F. Lapierre, and J. Flouquet, Phys. Rev. B 40, 10917 (1989); J.-M. Mignot, J. Flouquet, P. Haen, F. Lapierre, L. Puech, and J. Voiron, J. Magn. Magn. Mate. 76-77, 97 (1988), references therein.

9 K. Hanzawa, K. Ohara, and K. Yosida, J. Phys. Soc. Jpn. 66, L3001 (1997); K. Ohara, K. Hanzawa, and K. Yosida, ibid. 68, 521 (1999).

10 S. R. Saha, Ph. D. thesis, Tokyo Metropolitan University, 2001.

11 Y. Uwatoko, J. L. Sarao, J. D. Thompson, N. Môri, and G. Oomi, Rev. High Pressure Sci. Technol. 7, 1508 (1998);
H. Mori, N. Takeshita, N. Môri, Y. Uwatoko, Physica B 259-261, 58 (1999).

12 L. D. Jennings and C. A. Swenson, Phys. Rev. 112, 31 (1958).

13 T. F. Smith and C. W. Chu, Phys. Rev. 159, 353 (1967).

14 D. Weber, Ph. D. thesis, Frankfurt University, 1991.

15 T. Endstra, G. J. Nieuwenhuys, and J. A. Mydosh, Phys. Rev. B 48, 9595 (1993).

16 H. Yamagami and A. Hasegawa, J. Phys. Soc. Jpn. 62, 592 (1993).

17 U. Walter, Z. Phys. B: Condens. Matter 62, 299 (1986).

18 H. Ikeda and K. Miyake, J. Phys. Soc. Jpn. 65, 1769 (1996). 